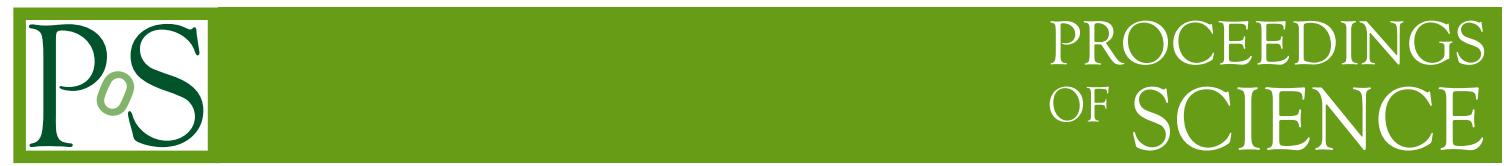

\title{
Overview of the CMS Strip and Pixel Detectors
}

\author{
Claude Patrick J Nuttens* \\ Universite Catholique de Louvain, Louvain-la-Neuve \\ E-mail: claude.nuttens deern.ch
}

The CMS silicon tracker is the largest silicon detector ever built. It's constituted of a 66 million channels pixel detector and a 10 million readout channels silicon strip detector with an active area of approximately to $200 \mathrm{~m}^{2}$. This document presents an overview of the operational experience gained during the last running period and the performance achieved including the impact on physics.

22nd International Workshop on Vertex Detectors

September 15th-20th, 2013

Lake Starnberg, Germany

\footnotetext{
* Speaker.

${ }^{\dagger}$ for the CMS collaboration, with FRIA-FNRS funding
} 


\section{Introduction}

The CMS tracking system [1] is composed of a silicon pixel and strip detector with optical analog readout. The pixel detector uses n-in-n silicon sensors with a pixel size of $100 \mu \mathrm{m} \times 150$ $\mu m$. Groups of $50 \times 80$ pixels are read by one read out chip. The barrel part (BPIX) is a combination of 3 concentric layers closed by two endcap (FPIX) disks on each side. It provides 66 million pixels for precise 2D measurements. It's surrounded by the silicon tracker, that represents with its 15148 individual silicon modules around $200 \mathrm{~m}^{2}$ of active area. The strip tracker p-in-n silicon sensors are arranged in 10 barrel layers and 3+9 endcap disks. They have a pitch range from $80 \mu \mathrm{m}$ for the inner layers up to $205 \mu \mathrm{m}$ for the outer ring endcaps and a 300(500) $\mu \mathrm{m}$ thickness in the inner(outer) parts. Four barrel layers and three end-cap rings are equipped with stereo modules. Composed of two silicon modules mounted back-to-back with a $100 \mathrm{mrad}$ stereo angle, they provide, in the strip tracker, a $2 \mathrm{D}$ hit resolution. Figure 1 gives an overview of the structure in the rz view ${ }^{1}$ of one quarter of the CMS silicon tracker.

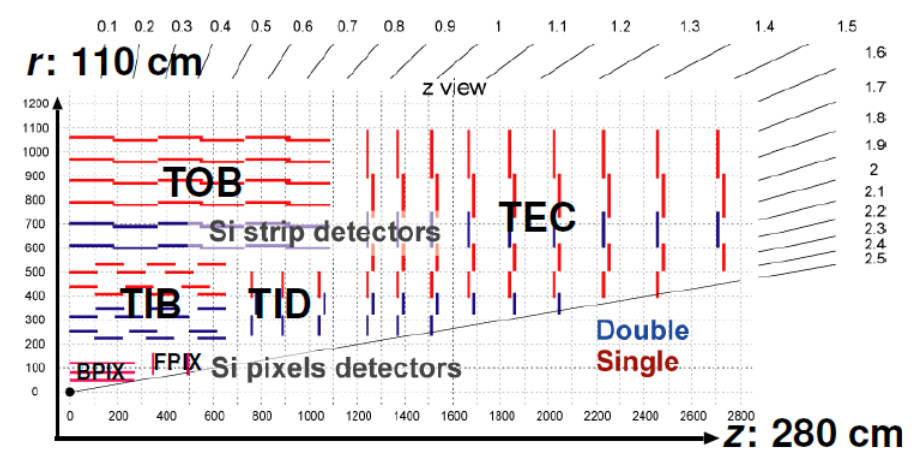

Figure 1: rz view of one quarter of the CMS silicon tracker.

During the last years of data taking, the silicon tracker has been in almost continuous operation. A good stability of the operational channels was observed. At the end of the data taking, in the pixel detector only around 2.3(7.2)\% of the BPIX (FPIX) channels could not be read and more than $97.5 \%$ of the strip tracker modules were still active.

As the LHC is aiming, in the following years, for an energy of $\sqrt{s}=13 \mathrm{TeV}$, with a $25 \mathrm{~ns}$ bunch spacing and a peak instantaneous luminosity of around $1.7 \times 10^{34} / \mathrm{cm}^{2} / \mathrm{s}$, work is done on the silicon detectors during the Long Shutdown (LS1) to get it ready. The pixel detector was extracted and stored in a clean room and broken parts were repaired: the BPIX (FPIX) will be brought back to $98.9 \%$ (100\% minus one chip). Two pixel pilot blades with modules from phase 1 upgrade will be installed and the pixel detector will then be centered precisely during its 2014 reinsertion. On the silicon strip detector, that can't be taken out, as many as possible accessible damaged parts will be recovered, and its temperature will be lowered down to $-10{ }^{\circ} \mathrm{C}$ on the sensors with a $-20{ }^{\circ} \mathrm{C}$ coolant to counteract the degradations from radiation.

\footnotetext{
${ }^{1} \mathrm{CMS}$ uses a right-handed coordinate system, with the origin at the nominal interaction point, the $\mathrm{x}$-axis pointing to the center of the LHC ring, the y-axis pointing up (perpendicular to the LHC plane), and the z-axis along the counterclockwise-beam direction. The polar angle, $\theta$, is measured from the positive z-axis, the azimuthal angle, $\phi$, is measured in the $\mathrm{x}-\mathrm{y}$ plane and the radial coordinate in this plane is denoted by $r$.
} 


\section{Tracker performance}

Both tracker subdetectors have been working successfully since their installation and provides excellent performance [3, 4].

\subsection{Hit efficiencies}

For the pixel detector, the hit efficiency is above $99 \%$ for all the layers as visible in figure 2. This efficiency, measured pixel-by-pixel, depends on the pile up, the L1 trigger rate and on the instantaneous luminosity due to the occupancy. An unavoidable dynamic inefficiency was observed, as seen in figure 3 , which increases with hit rate and originates mainly from limitations in the internal buffering of the readout chip [2].

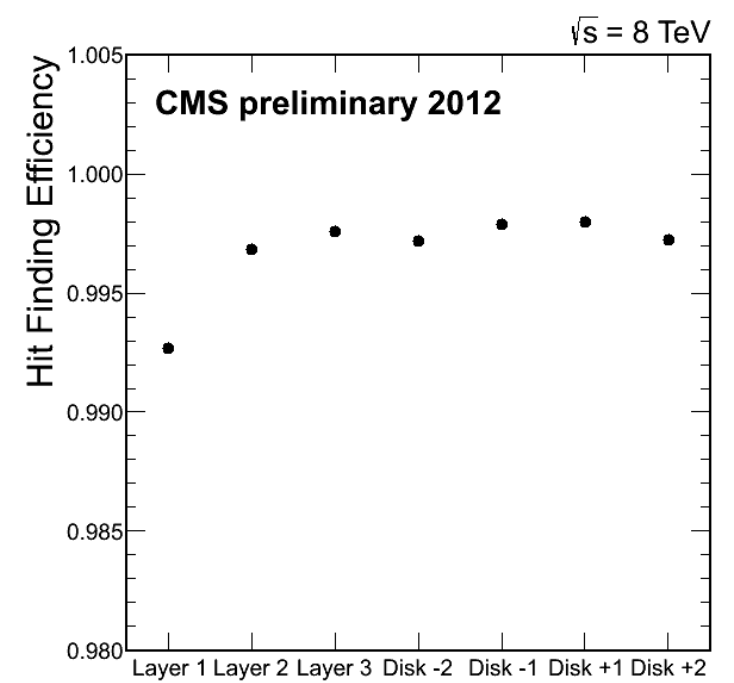

Figure 2: Hit efficiency by layers for the pixel detector.

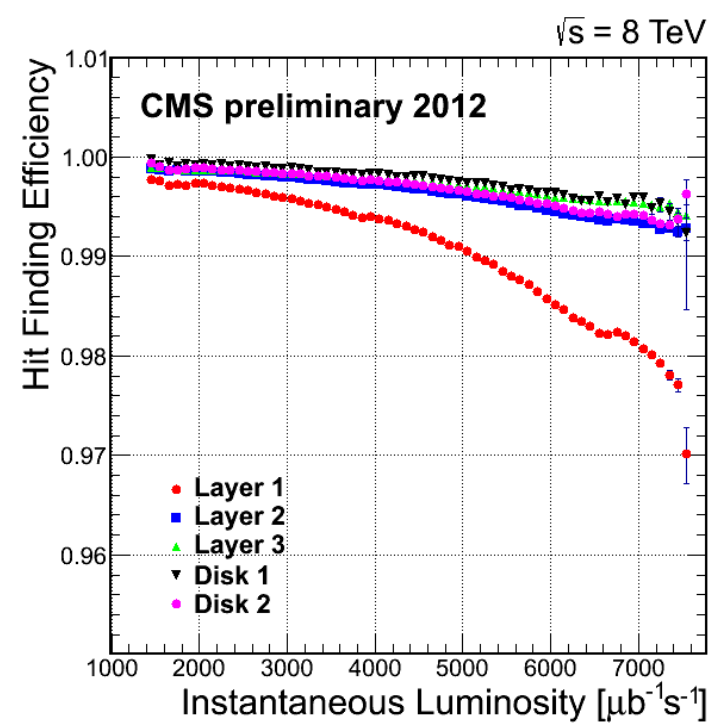

Figure 3: Hit finding efficiency dependence on the instantaneous luminosity for the pixel detector.

On the strip detector side, it was looked at the signal-to-noise ratio of hits on reconstructed tracks, where the ratio is corrected for the track angle with respect to the sensor surface to take the path length into account. As shown on figure 5, the distributions nicely follow a Landau distribution convoluted with Gaussian resolution that gives the most probable value (MPV) of 18.4(22.4) for the inner barrel, TIB (outer barrel, TOB). The difference comes from the sensors thickness and pitch difference between the module types. Those values are in good agreement with values obtained after irradiation campaigns prior to the detector integration [5]. This allows for a very good hit efficiency of more than $92 \%$ for all the modules, as figure 4 shows. Taking into account the inefficient regions, the hit efficiency goes up to about $99.8 \%$.

\subsection{Pixel and strip detectors resolution}

The resolution of the subdetectors is studied using collision data. The hit resolution in the pixel detector can be determined using the triplet method: tracks with hits in the three barrel layers 


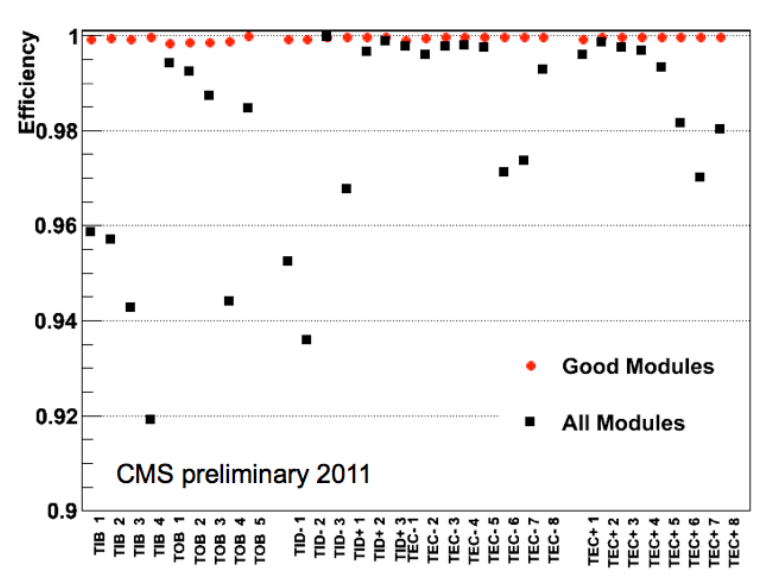

Figure 4: Strip detector hit efficiency by detector region.

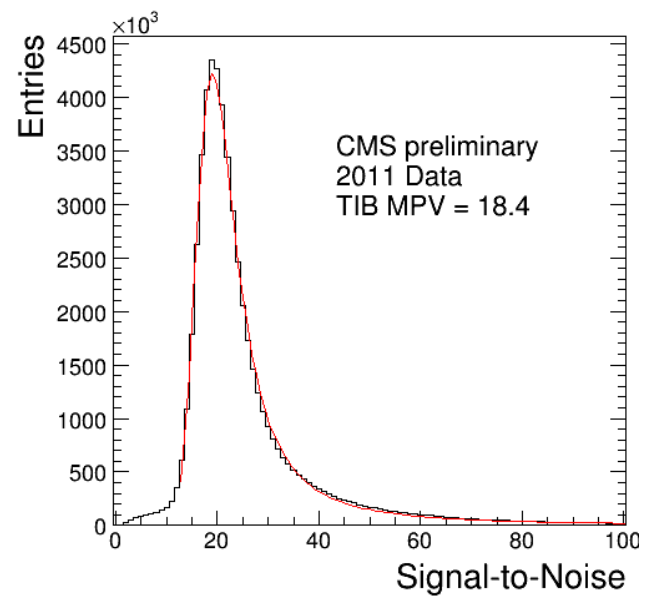

Figure 5: Strip detector signal to noise ratio in TIB.

are refitted without the hit in the layer 2 using the layers 1 and 3 positions and angles. Then the residuals difference between the hit position and the interpolated track can be determined. They are plotted in figures 6 and 7. An intrinsic resolution can then be determined assuming the resolution is the same in all three layers.

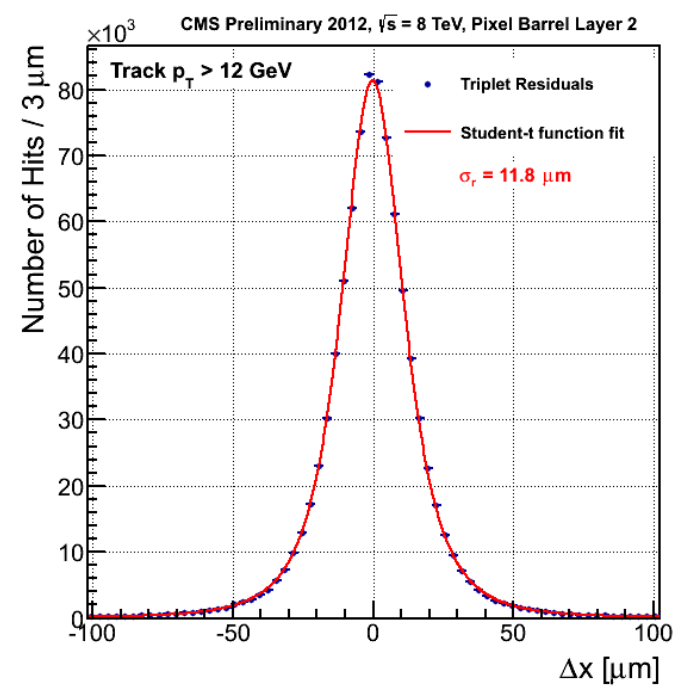

Figure 6: Pixel triplet $\mathrm{x}$-resolution determined by taking the residual difference between the hit position and an interpolated track in layer 2

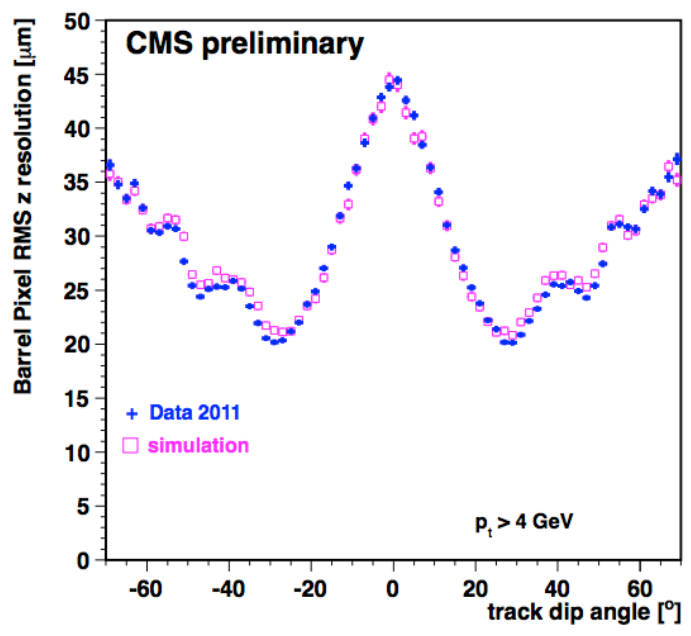

Figure 7: Pixel resolution in $\mathrm{z}$ direction.

\subsection{Momentum, vertexing and $b$-tagging performance}

The good hit resolution in the pixel and strip trackers and the many measurement points per track, lead to a very good momentum resolution $(\sigma(p t) / p t=1-2 \%$ for a muon with a transverse 
momentum of $100 \mathrm{GeV} / \mathrm{c}$ ) using only the tracker. Those good resolutions allow to have a very good primary vertex resolution as shown in figure 8 . Such performance provides precise and very discriminant inputs for the b-tagging, as for example the 3D impact parameter uncertainty shown in figure 9. In this figure, it is also shown that the performance is well described by the simulation.

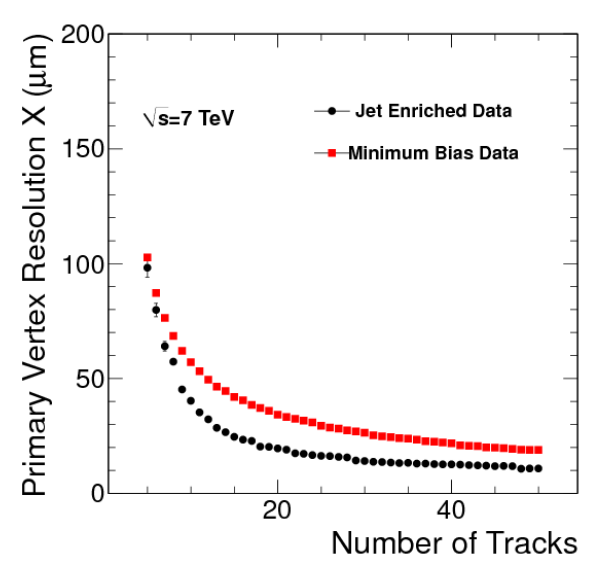

Figure 8: Primary vertex $\mathrm{x}$ resolution with respect to the number of tracks in the vertex.

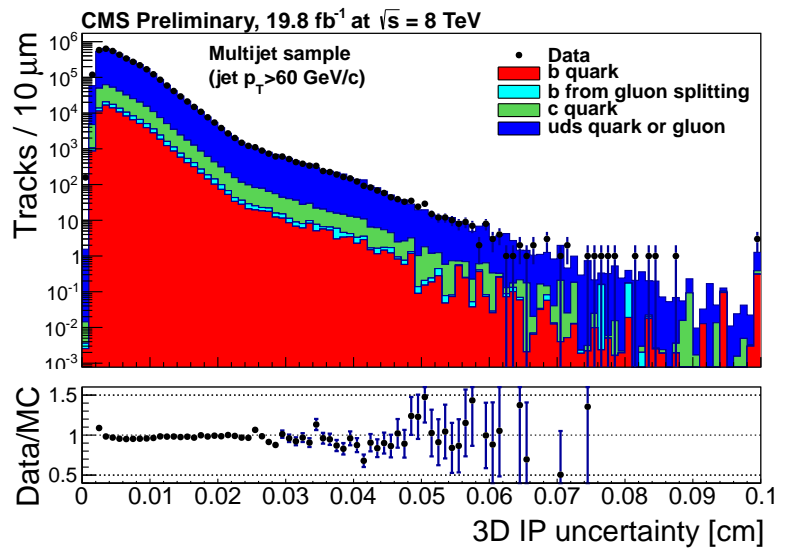

Figure 9: 3D impact parameter uncertainty on data and MC.

\subsection{Time and spatial alignment}

This performance is achievable because of a good time and spatial alignment of the detector [6]. For example, the signal time profile in the strip tracker has a great impact on the signal-to-noise ratio if the timing is off. The efficiency will suffer if the timing is far off and this also impacts the occupancy due to the out-of-time pile up (OOP). As visible in figure 10, the time profile of all subsystems is centered correctly. It was also noticed to be stable with time. The impact in $50 \mathrm{~ns}$ bunch spacing running is minor, but the control and stability on the timing is essential at $25 \mathrm{~ns}$ as a few nanosecond delay leads to two up to ten times more OOP.

The track-based alignment is working so well it is now sensitive to the sensor curvature. The curvature of single-sided sensors is revealed in figure 11, where the average track angle corrected residuals is plotted versus the local direction transverse to the strip $\mathrm{u}\left(\langle\Delta W\rangle=\left\langle\left(u_{t r k}-u_{h i t}\right) / \tan \psi\right\rangle\right.$, with $\psi$ the track angle with respect to the sensor surface) . A sensor-by-sensor correction is applied, so that the module can be treated as flat in the tracking software.

\section{Detector evolution with integrated luminosity}

The tracker subdetectors are designed to still operate after the significant radiation damage sustained during the first years of running at 7 and $8 \mathrm{TeV}$. A monitoring of the evolution of the damage is done and compared with simulations and expectations. This will be presented below including studies of the evolution of the leakage current, the bias voltage, the pixel threshold, the cluster charge, and finally the Lorentz angle. 


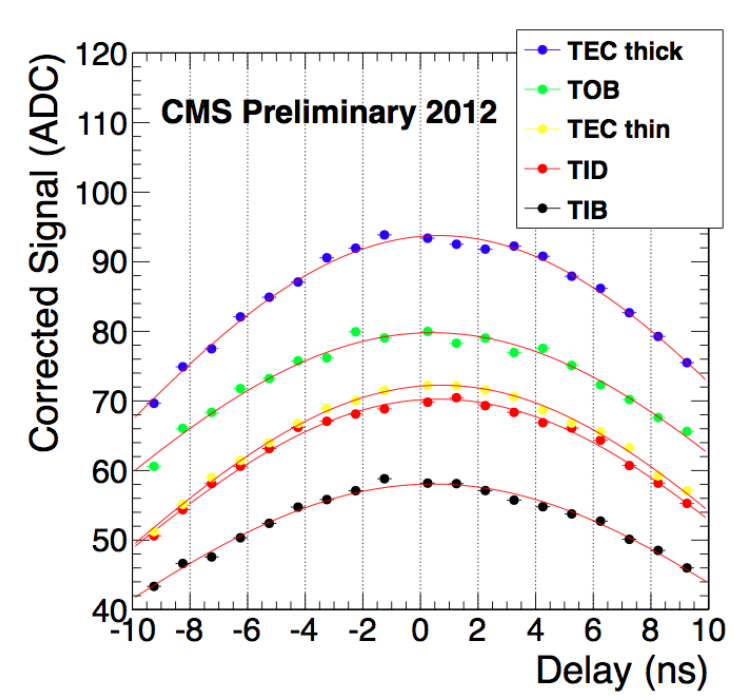

Figure 10: Signal time profile for the various Strip detector regions.

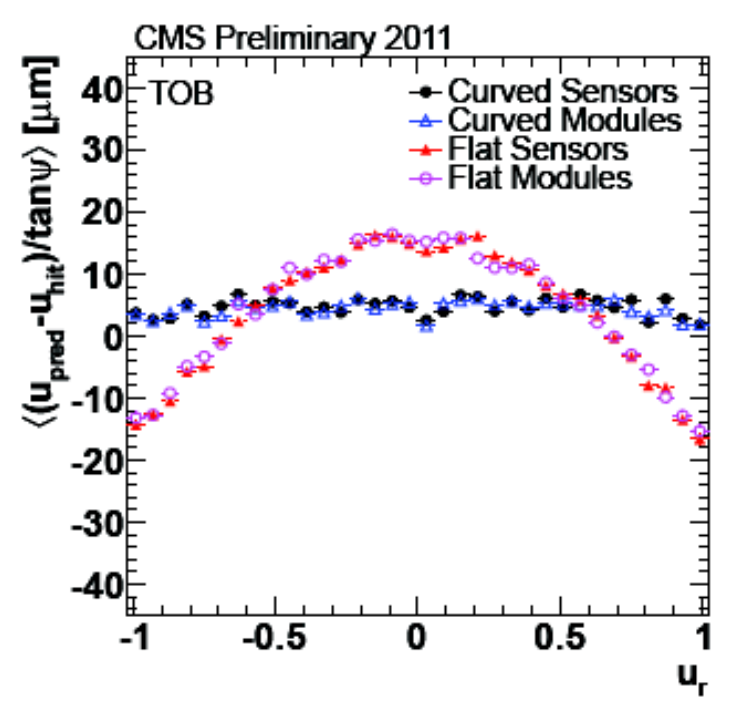

Figure 11: Average track angle corrected residuals vs module strips transverse direction.

\subsection{Leakage currents}

Using the readings from the high voltage power supplies, the leakage current in the pixel tracker barrel is measured and compared with the evolution models with respect to the radiation damage. The leakage current is expected to increase linearly with fluence due to bulk silicon damage although radiation damage can partially be recovered due to annealing. This trend is indeed observed in figure 12 where the leakage current is represented with respect to the integrated luminosity. In figure 13, the data are compared to a parametrization[7] adding exponential and logarithmic terms accounting for the accumulated damage and the annealing whose input is the fluence predicted by a CMS detector model implemented in FLUKA[8, 9]. A good shape agreement with the models is noticed but a positive rescaling of the normalization by $40-70 \%$ is required. The reason for this discrepancies in scale are under investigation, the uncertainties in the operational temperature of the detector could be a possibility. Also, an azimuthal dependence of the leakage current is observed, which arises from the fact that the LHC beam spot is not perfectly at the center of the pixel detector, and could lead to a $30 \%$ effect on the potential layer 1 lifetime. This is expected to be fixed after the LS1 pixel detector re-centering.

Concerning the strip detector, the leakage current is also expected and observed to be increasing with the fluence. This gives a handle to measure the effect of the accumulated irradiation, for example the leakage current that can be measured module-by-module using the detector control units (DCUs) and current measurements from the power supply units. In average, the strip tracker measurements agree with the models (FLUKA [8, 9]) within 5-20\%, as seen on figure 14 where the leakage current measured in strip detector is compared to the simulated one and corrected for module type, radius, and sensor expected temperature. It is observed that the leakage current is varying over time and detector region as it is temperature dependent and the actual temperature of the silicon tracker is not uniform. Consequently, the agreement is better in detector regions with 


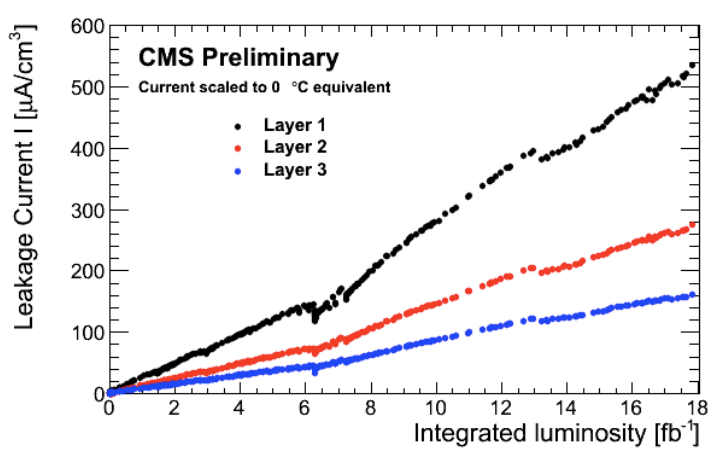

Figure 12: Average leakage currents in each pixel barrel layer as a function of integrated luminosity.

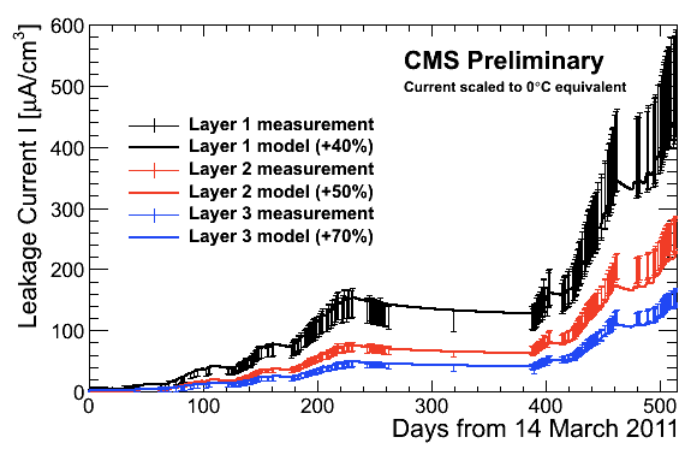

Figure 13: Average leakage currents in each pixel barrel layer as a function of time.

temperature lower than $20^{\circ} \mathrm{C}$.

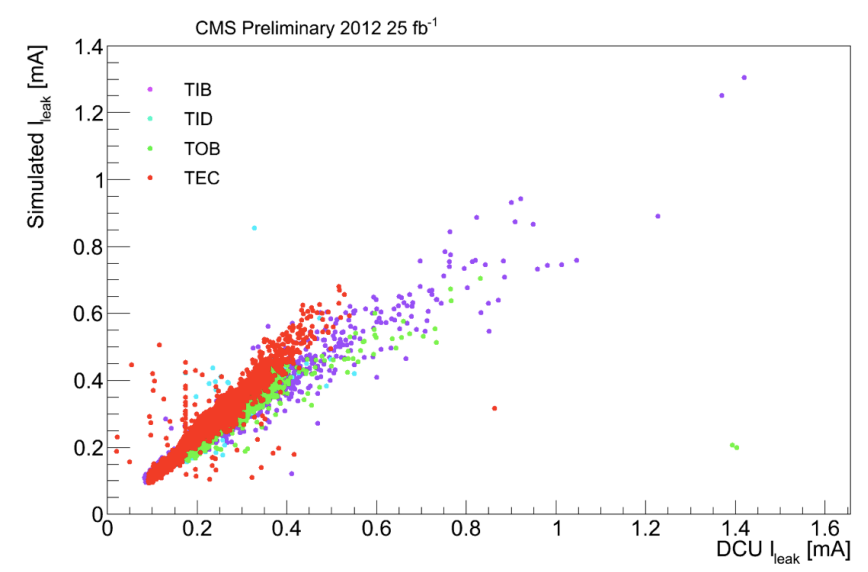

Figure 14: Strip detector leakage current in data compared to simulation, corrected for module type, radius and sensor temperature.

\subsection{Depletion voltage in the pixel detector}

To investigate depletion voltage evolution in the pixel tracker, regular measurements of the hit efficiency have been done during bias voltage scans, changing the charge collection and thus the hit efficiency. The results of the bias scans for the pixel layer 1 are shown in figure 15. In figure 16 , the bias voltage needed in order to reach a $99 \%$ efficiency at various integrated luminosities is plotted. In this figure, layer 1 and 2 reach a minimum at around $10 \mathrm{fb}^{-1}$ of integrated luminosity, showing that those layers have been type inverted during the 2012 data taking.

\subsection{Pixel thresholds and cluster charge}

During the data taking, the minimum pixel threshold required to not affect the data quality was increased, as shown in figure 17. This required optimizations done first in 2011 for the complete pixel detector and again in 2012 for the barrel. An explanation to this increase could be that bulk 


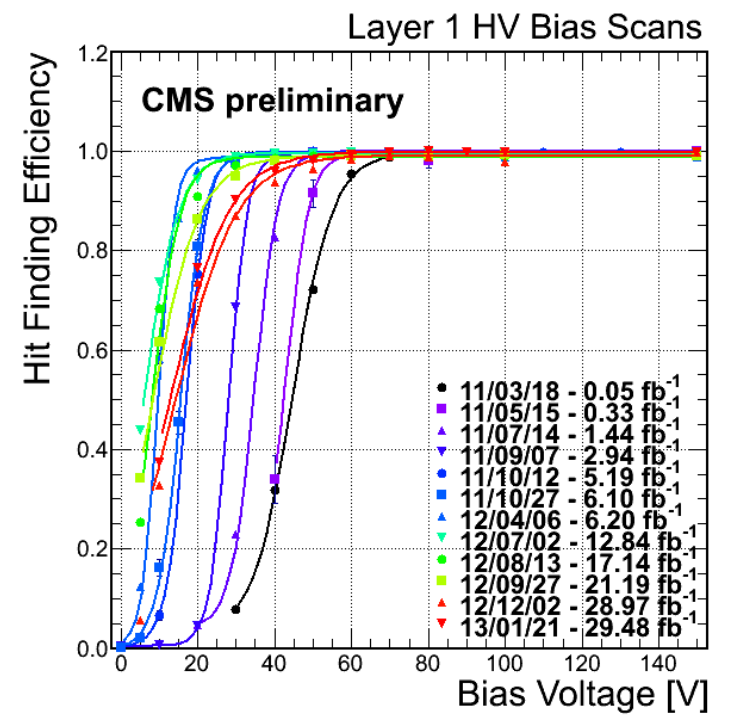

Figure 15: Hit efficiency results from the bias scan, at various luminosities, for layer 1.

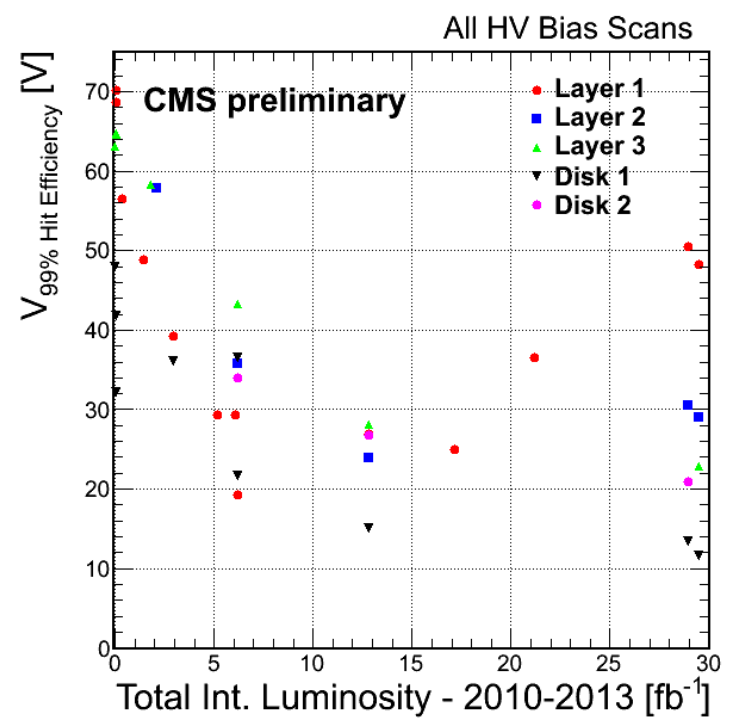

Figure 16: Voltage to reach $99 \%$ hit efficiency at different integrated luminosities.

damage in a diode used as a reference voltage within the ROC caused a change in the meaning of the DAC settings.

There have also been studies on the stability of the cluster charge. For each layer in the pixel tracker barrel, the cluster charge distribution is fitted by a Landau convoluted with a Gaussian, from which the MPV is extracted. The procedure is done at various integrated luminosities and plotted in figure 18. The cluster charge is observed to have a stable behavior with the integrated luminosity.

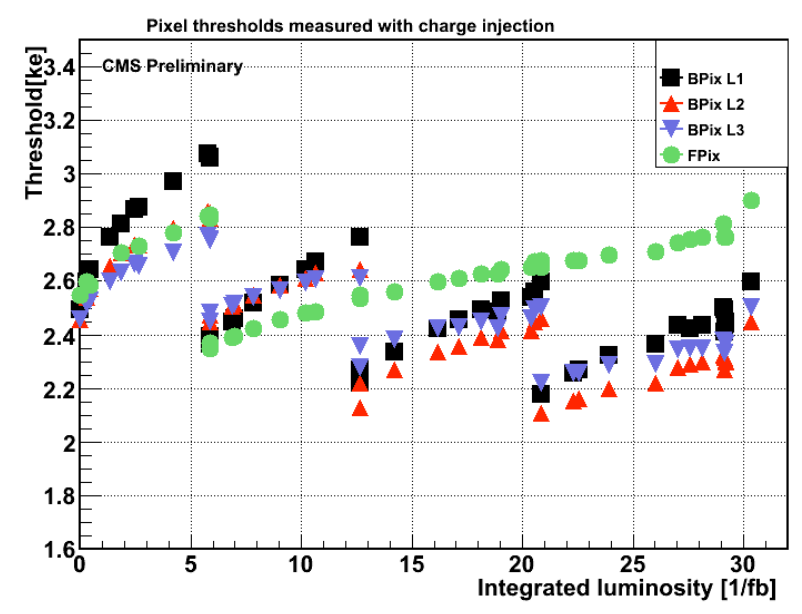

Figure 17: Pixel thresholds as a function of the integrated luminosity. Optimizations were done in 2010 for the whole detector and for barrel layers in 2012.

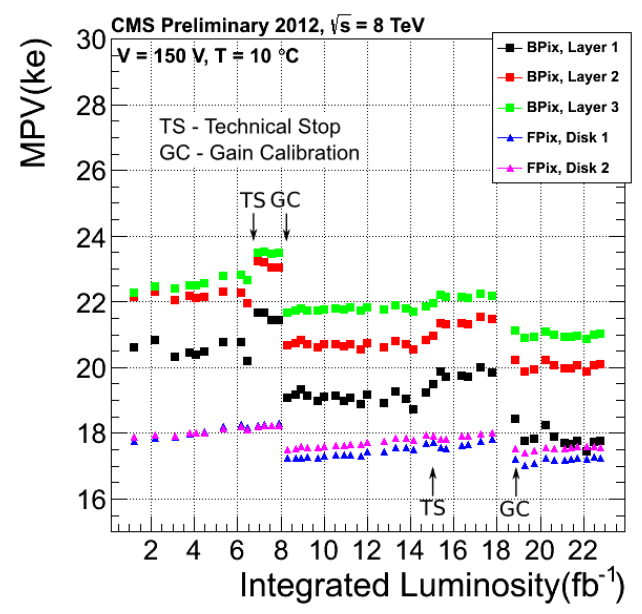

Figure 18: MPV extracted from cluster charge fit for various integrated luminosities. 


\subsection{Lorentz angle in the pixel tracker}

Finally, the evolution of the Lorentz angle with the integrated luminosity was studied. The Lorentz angle value is increasing with the integrated luminosity and is determined using various techniques: the grazing angle method and through the alignment. The first method determines the charge carrier production depth and displacement to compute the drift distance with respect to the production depth and extract the Lorentz angle. The second one uses the alignment tools (MILLIPEDE) and the tracks collected when the magnet was in on and off modes to determine the drift caused by the magnetic field. The evolution of the Lorentz angle is shown in figure 19 for the grazing angle method, and in figure 20 using the alignment. The irradiation affects both techniques in a different way. The alignment method provide the effective value of the Lorentz angle when the grazing method is more sensitive to the value in the center of the sensor. Before irradiation, the two values are close to each other as it is roughly stable in the sensor and equal to the value in the center. After irradiation of the sensor, it is not the case anymore. One effect visible in figure 20 and still needs to be better understood is the offset between $\mathrm{z}+$ and $\mathrm{z}$ - side of the detector. Indeed, for all the rings of modules at the same z-distance in layer two, the ones at positive values in the z-direction have a bigger $\tan \left(\theta_{L A}\right)$ than the ones at negative values. Different operating conditions in both direction could be a possibility. As seen on the plots, different shape of evolution among layers are observed, and still need to be deeper undersood.

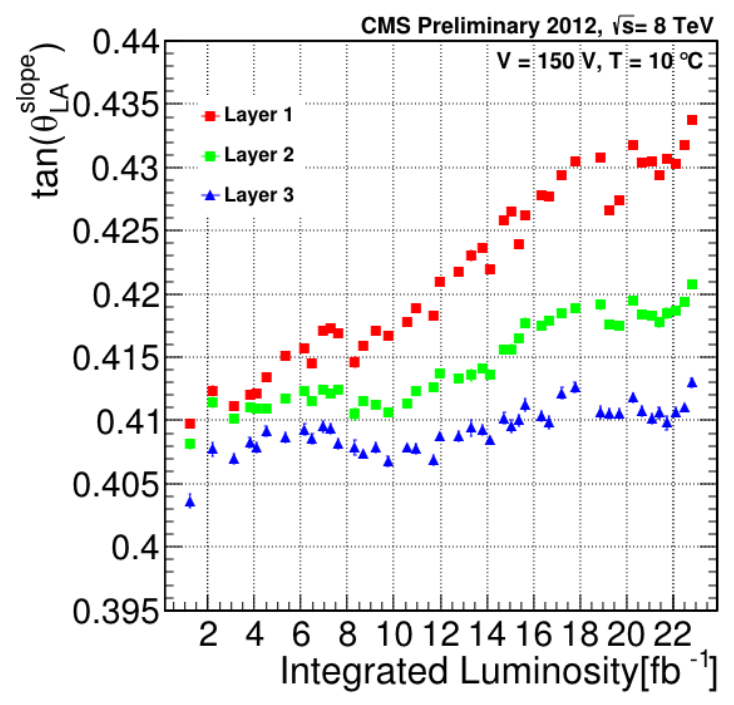

Figure 19: Lorentz angle evolution using the grazing angle method.

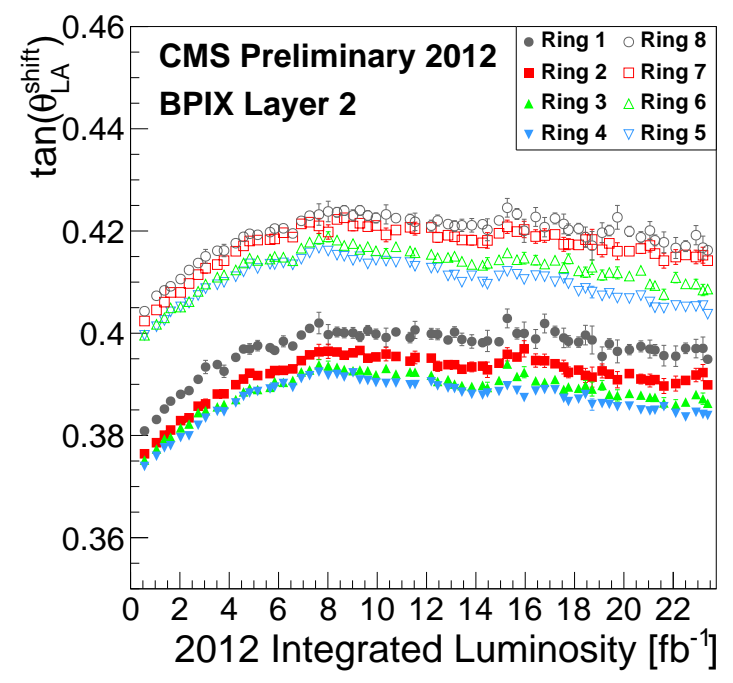

Figure 20: Lorentz angle evolution using the alignment.

\section{Conclusions}

The CMS tracker worked well during the past years of data taking. Even if some channels were lost, they are mainly (100\% minus one chip) expected to be recovered after LS1 in the pixel tracker. In the strip tracker, the lost channels (about 2.5\%) are less problematic given the high number of layers. As presented here, the detector has excellent performance, in signal-to-noise 
ratio, detection efficiency, in spatial, momentum and vertexing resolution, etc. This was achieved notably because of a good alignment (sensitive up to the sensor curvature in the strip detector) and timing control. Also, the aging of the detector is monitored, compared with predictions and work is done to understand the discrepancies. This gives a good knowledge of the status of the detector before the restart of the LHC after the LS1 during which work is done for a full recovery of the pixel detector and the lowering of the temperature in the strip detector.

\section{References}

[1] S. Chatrchyan et al. [CMS Collaboration], The CMS experiment at the CERN LHC, JINST 3 (2008) S08004.

[2] CMS Collaboration, CMS technical design report for the pixel detector upgrade, CERN, Geneva, Tech. Rep. CERN-LHCC-2012-016. CMS-TDR-011, Sep 2012. Available online at: http://cdsweb.cern.ch/record/1481838.

[3] CMS Collaboration, Commissioning and performance of the CMS silicon strip tracker with cosmic ray muons, 2010 JINST 5 T03008, March 2010, [physics . ins-det / 09114996 ]

[4] CMS Collaboration, CMS tracking performance results from early LHC operation, Eur. Phys. J. C (2010) 70: 1165-1192, Nov 2010. [physics.ins-det/10071988]

[5] C. Eklund, A. Heikkinen, A. Honkanen, V. Karimäki, T. Mäenpää, E. Pietarinen, H. Saarikoski, K. Skog, J. Tuominiemi, T. Tuuva, Silicon beam telescope for CMS detector tests, Nuclear Instruments and Methods in Physics Research A , 430 (321-332), Jul. 1999

[6] CMS Collaboration, Alignment of the CMS silicon tracker during commissioning with cosmic rays ,2010 JINST 5 T03009, March 2010, [physics.ins-det/ 09102505 ]

[7] M. Moll, Ph.D thesis, University of Hamburg (1999), DESY-THESIS-1999-040. Available at http://www-library.desy.de/diss99.html.

[8] A. Ferrari, P. R. Sala, A. Fasso and J. Ranft, FLUKA: A multi-particle transport code (Program version 2005), CERN-2005-010.

[9] G. Battistoni, S. Muraro, P. R. Sala, F. Cerutti, A. Ferrari, S. Roesler, A. Fasso and J. Ranft, The FLUKA code: Description and benchmarking, AIP Conf. Proc. 896 (2007) 31. 